\title{
An Automatic Surface Defect Detection System for Hot Ingot Casting Slabs Using an Infrared Scanning Camera and Image Processors*
}

\author{
By Hajime KITAGAWA,** Akira FUJII,** Shigeru MIYAKE** \\ and Kunio KURIT $A^{* *}$
}

\begin{abstract}
Synopsis
An automatic surface defect detection system for hot ingot-casting-slabs has been developed to support hot-direct-rolling operations. Among several possibilities considered, the thermal image of a hot slab provided by an infrared scanning camera was evaluated as being the best. Having shown that the majority of surface defects could be recognized visually on the thermal image, an image processing system to replace visual recognition was developed. The image processing system as designed consists of a signal processing unit and a mini-computer. Several different data processing stages are involved in the extraction of defect signals. Many parameters in the processing system were given a range of freedom and the final values adjusted to provide optimum detectability for actual defects. It was concluded that this detection system would enable on-line defect detection if some minor improvements were made. Two topics now under consideration are the coupling of the detection system to an automated spot scarfer and the application of the system to defect detection in continuous-castingslabs.
\end{abstract}

\section{Preface}

Hot-direct-rolling, in which the ingot-casting-slab is conveyed directly to a hot rolling mill, skipping an intermediate stage of cooling and reheating the slab, is one of the most likely energy saving techniques for steel making processes. The operation of such a hotdirect-rolling process requires the detection and removal of defects which appear on the surface of the hot slab.

This paper focuses on the development of a detection system which automatically detects surface defects on a " hot slab", with reference to the coupling of the system to an automated reconditioning machine (a spot scarfer). (Note: A " hot slab" means in this paper a slab at the high temperature of a soaking pit, that is, a slab above $1000^{\circ} \mathrm{C}$.) In addition, it also considers the application of this system to surface defect detection in a continuous-casting-slab. The detection and removal of such defects is also important in the operation of hot-charge-rolling, which is analogous to hot-direct-rolling in that in hot-chargerolling a continuous-castng-slab is conveyed directly to a reheating furnace, skipping the slab cooling stage.

\section{Flow Diagram for Hot-direct-rolling and Hot- charge-rolling}

Figure 1 shows a possible flow diagram in which every slab experiences either hot-direct-rolling or hotcharge-rolling. The 'Defect Detector' in Fig. 1 corresponds to the present detecting system. As seen in Fig. 1, a continuous-casting-slab is supposed to be inspected after being discharged from the reheating furnace, and an ingot-casting-slab after being crop- sheared. One of the advantages of such parallel inspection of both kinds of slabs is that both can share a common defect detector.

\section{Double Inspection of a Continuous-casting-slab}

As shown in Fig. 1, a continuous-casting-slab, unlike an ingot-casting-slab, is supposed to undergo a primary inspection just after the slab is cast. The idea of this primary inspection derives from the generally-accepted theory that defect information should be fed back to the preceding operation to reduce defects, if the operation can make use of this information and, in addition, there is the space for such an inspection.

The authors originally selected as the best position for the primary inspection the vicinity of an upper roller apron, but it turned out that the image of a continuous-casting-slab taken at this point was too poor to be able to distinguish oscillation marks from cracks stretching in the transverse direction. It now would seem better to shift the inspection site to another point, for example to the delivery side of the pinch rolls. With an increase in continuous-casting-slab production, not only a primary inspection but also a primary reconditioning might become necessary.

\section{Defect Detection System}

\section{Alternative Components for a Detecting System}

There are a number of alternative components which can be used in a defect detection system, as shown in Fig. 2 and described below:

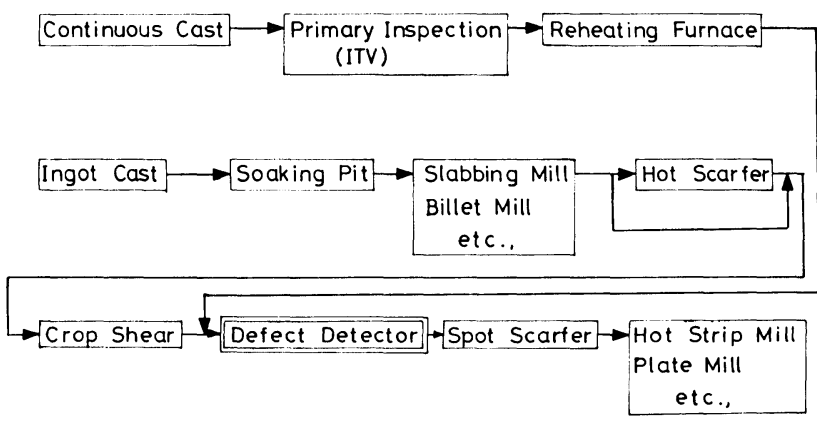

Fig. 1. Flow sheet of hot-direct-rolling and hot-chargerolling.

A continuous-casting-slab is primarily inspected by, for example, an industrial television camera (ITV) and then inspected by a "Defect Detector". An ingot-casting-slab is inspected by the same detector after crop-shearing.

\footnotetext{
* Partly presented at the 96th ISIJ Meeting, October 1978, and at the 97th ISIJ Meeting, April 1979. Manuscript received December 20, 1979.
}

** Research Laboratories, Kawasaki Steel Corporation, Kawasaki-cho, Chiba 260. 


\section{(1) Optical or Non-optical Methods}

A typical example of a non-optical method would be the probe-type eddy current method, which is being developed by Sumitomo Metal Industries, Ltd. (Japan), Förster Co. (Germany) and Bergstrand Co. (Sweden). Optical methods are divided into two groups depending on the wavelength used, infrared or visible.

(2) Active or Passive Methods

An active method is one which makes use of the reflection of radiation directed at an object. A passive method is one which makes use of the radiation from the object itself.

(3) Scanning or Imaging Methods

A scanning method obtains surface information by some kind of scanning action, using, for example, a rotating mirror or a shift register. An imaging method forms an image of the field of view with lenses.

(4) Image Display or Other Methods

According to the degree of complexity, the presentation of defect information can be divided into three types.

1) Visual monitoring of the image on a TV screen,

2) Evaluation and output of defect information through a data processor, and

3) Conveying defect information to a reconditioning machine, to enable on-line detection and removal of surface defects.

\section{Specification of the Detection System}

From among the several possibilities listed in Fig. 2 , the authors selected the following combination for their detection system: An infrared scanning camera senses the radiation from a hot slab (passive). Image processing equipment detects surface defects by processing the data from the scanning camera. The processing equipment then conveys the defect information to a spot scarfer.

The items underlined in Fig. 2 correspond to this system. Their selection was made for the following reasons:

(1) The spectrum radiated from a hot slab has its peak in the infrared range.

(2) The radiation from a hot slab is expected to vary with the presence of surface defects (passive).

(3) For a body moving at a constant low speed, the simplest way to obtain complete surface information is the compiling of data obtained by (transverse) scanning perpendicular to the direction of motion at a fixed longitudinal position.

(1) Optical Method.

$\left[\begin{array}{l}\text { Infra-red } \\ \text { Visible Range }\end{array} \quad \begin{array}{l}\text { Active Method } \\ \text { Passive Method }\end{array} \quad\left[\frac{\text { Scanning Method }}{\text { Image Making Method }}\right.\right.$

Image Display

- Data Processing, Defect Deta Output

Combining Spot Scarfer with Defect Detector

(2) Other Method

e.g. Ultra-Sonic Method, Eddy Current Method.

Fig. 2. Alternative items of a surface defect detection system.
(4) The coupling of the detector to a sport scarfer is most desirable from the point of view of labor efficiency.

\section{Infrared Scanning Camera}

\section{Specifications of the Detector Size and the Filter}

Figure 3 shows the optical path of the scanning camera, which is equipped with two detectors, a large one and a small one, and four filters. The large detector, having a better temperature resolution but a worse spacial resolution of $4 \mathrm{~mm} \times 4 \mathrm{~mm}$ at the slab surface is arranged to detect relatively large defects, such as scabs, while the small detector, having a worse temperature resolution but a better spacial resolution of $1 \mathrm{~mm} \times 1 \mathrm{~mm}$, is used to detect relatively small or thin defects, such as longitudinal cracks or blow-holes. Filter 1 divides the incident energy equally between the two detectors. Filter 2 partitions the energy so that wavelengths from 1.0 to $2.4 \mu \mathrm{m}$ are sent to the small detector and wavelengths from 2.4 to $4.0 \mu \mathrm{m}$ to the large detector. In other words, Filter 2 directs the higher temperature radiation mostly to the small detector and the lower temperature radiation mostly to the large detector. This filter was based on two considerations :

(1) The radiation from concave defects, such as cracks or holes, which usually have a higher temperature than normal areas and are often as large as $1 \mathrm{~mm} \times 1 \mathrm{~mm}$, should be directed to the small detector, which has a finer spacial resolution.

(2) The radiation from other defects, such as scabs, which usually have a lower temperature and are often larger than $4 \mathrm{~mm} \times 4 \mathrm{~mm}$, should be sent to the large detector.

Filter 3 directs all the energy to the large detector and Filter 4 directs it to the small detector.

A number of possible combinations of detectors and filters were compared in terms of defect-detectability. "Thin crack" was simulated in the laboratory for this comparison. The crack was made up of two lengths of wire with different diameters and different

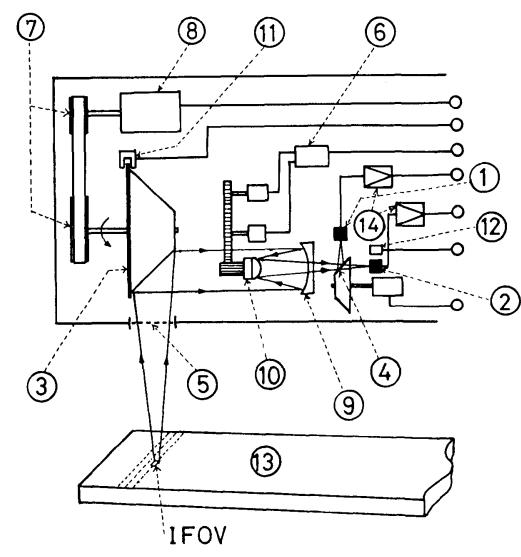

(1) Detector: Large FOV (2) Detector: Small Fov

(3) Scanning Mirror

(4) Optical Filter

(5) Window

(6) Focus Control

(7) Pulley

(8) Scanning Motor

(9) 1'st Lens

(10) 2 'nd Lens

(11) Chopper

(12) Thermistor

(13) Hot Slab

(14) Signal Amplifier

Fig. 3. Optical alignment of the infrared scanning camera. A part of a slab surface, marked as IFOV (instant field of view), is now being imaged both on a detector with large FOV (field of view) and a detector with small FOV. 
temperatures; one of $0.3 \mathrm{~mm}$ diameter at $750^{\circ} \mathrm{C}$ and the other of $8 \mathrm{~mm}$ at $800^{\circ} \mathrm{C}$. The thinner wire $(0.3 \mathrm{~mm})$ was placed just in front of and parallel to the thicker wire and both were held vertically, so that the thicker wire looked as if it had a very thin $(0.3 \mathrm{~mm})$ dark $\left(50^{\circ} \mathrm{C}\right.$ lower temperature) crack along it axis. The infrared scanning camera scanned the pseudo-crack with various combinations of detectors and filters and gave an intensity distribution along the scanning direction, as shown in Fig. 4. Since defect-detectability was evaluated in terms of the smallness of a detectable defect, the combination of Filter 4 and the small detector was evaluated as best. As a result the infrared scanning camera was finally specified as listed in Table 1.

\section{Visual Inspection of Thermal Images Provided by the Infrared Scanning Camera}

The scanning camera, installed just behind a hot scarfer of the No. 2 slabbing mill at Chiba Works, collected data (thermal images) from more than a hundred hot slabs, most of which were at the state just after hot-scarfing and the rest of which had not been hot-scarfed at all (the slab surface just after hotscarfing is free of scale, although this soon appears on the surface). The slabs were then given a careful and minute (visual) inspection after cooling to ambient temperature. When a surface defect was found on a cooled slab, the information about the defect, such as the size, the location and the shape and a $35 \mathrm{~mm}$ photograph were collected. This information, especially the photographic image, was collated with the corresponding thermal image retrieved from a video tape recorder (VTR), and it was decided whether the defect could be visually recognized on
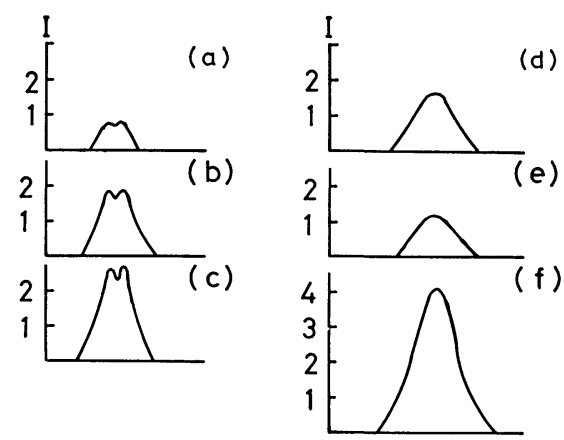

\begin{tabular}{l|l|cccccc}
\hline $\begin{array}{c}\text { Combi- } \\
\text { nation }\end{array}$ & Filter & 1 & 2 & 4 & 1 & 1 & 1 \\
\cline { 2 - 7 } & Detector & Small & Small & Small & Large & Large & Large \\
\hline $\begin{array}{l}\text { Resultant } \\
\text { intensity } \\
\text { distribution }\end{array}$ & (a) & (b) & (c) & (d) & (e) & (f) \\
\hline
\end{tabular}

Fig. 4. Detectability of a pseudo thin crack through various combinations of filters and detectors.

Each figure shows the intensity distribution of the crack image along the direction right to its axis. The combination of Filter 4 and the small detector, (c), is determined to give the best detectability because the crack, located at the center of each profile, is observed best in (c). (The unit of both axes in the figure is chosen arbitrary.) the thermal image. These inspection steps are listed in Fig. 5. Photograph 1 shows how a defect looks while the slab is hot and when it is cold. In this figure, "Cold Slab" shows a photograph of scabs found on a cold slab. White circles were added to mark the scabs. "Thermal Image" shows a thermal image of the same defects made while the slab was hot (just after hot-scarfing). The spots appearing on the upper left of the thermal image were determined to be defects from the good agreement with the scabs on "Cold Slab" in terms of location, shape and size. "Hot Slab" shows a photographic image of the same defects taken by a $35 \mathrm{~mm}$ camera while the slab was hot. Since the "Hot Slab" defect-image is generally more similar to the thermal image than

Table 1. Specifications of the infrared scanning camera.

\begin{tabular}{|c|c|c|}
\hline Items & Specification & Memorandom \\
\hline $\begin{array}{l}\text { Temperature range } \\
\text { to be measured }\end{array}$ & $700^{\circ} \sim 1400^{\circ} \mathrm{C}$ & \\
\hline $\begin{array}{l}\text { Temperature } \\
\text { resolution }\end{array}$ & $1^{\circ} \mathrm{C}$ & at $1100^{\circ} \mathrm{G}$ \\
\hline $\begin{array}{l}\text { Instantaneous field } \\
\text { of view (Spacial } \\
\text { resolution) }\end{array}$ & 0.4 mrad. $(1 \mathrm{~mm})$ & $\begin{array}{l}\text { (at a distance } \\
\text { of } 2.1 \mathrm{~m} \text { ) }\end{array}$ \\
\hline Field of view & $\pm 26.5^{\circ}$ & \\
\hline Detector & $\mathrm{HgCdTe}^{1)}$ & $\begin{array}{l}\text { Special cool- } \\
\text { ing }^{2)}\end{array}$ \\
\hline Scanning rate & $180 \mathrm{~Hz}( \pm 5 \%)$ & \\
\hline Focusing range & $2.0 \sim 2.2 \mathrm{~m}$ & \\
\hline $\begin{array}{l}\text { Presentation of } \\
\text { signals }\end{array}$ & $\begin{array}{l}\text { Intensity modula- } \\
\text { tion or waveform }\end{array}$ & \\
\hline Frequency band & $500 \mathrm{KHz}$ & \\
\hline Record of signals & $\begin{array}{l}\text { Video Tape } \\
\text { Recorder (VTR) or } \\
\text { photographic film }{ }^{3)}\end{array}$ & \\
\hline \multicolumn{3}{|l|}{ SLAB } \\
\hline $\begin{array}{l}\text { Usual conveyance } \\
\text { speed }\end{array}$ & $10 \mathrm{~m} / \mathrm{min}$ & \\
\hline $\begin{array}{l}\text { Surface being } \\
\text { inspected }\end{array}$ & upper surface & \\
\hline \multirow[t]{2}{*}{ Usual dimensions } & $\begin{array}{ll}\text { width : less than } \\
2 \mathrm{~m}\end{array}$ & \\
\hline & $\begin{array}{c}\text { length: less than } \\
10 \mathrm{~m}\end{array}$ & \\
\hline
\end{tabular}

1) HgCdTe: The spectral response of HgCdTe was closest to the spectral radiance from a hot slab as far as was checked.

2) Special cooling of the detector: The conventional way of keeping the detector at a constant temperature, that is the occasional supplying of liquid nitrogen, was regarded as inconvenient in automatic detection. Instead, an electric device was adopted.

3) Photographic film: All data can be recorded on photographic film as a waveform using a high speed camera.

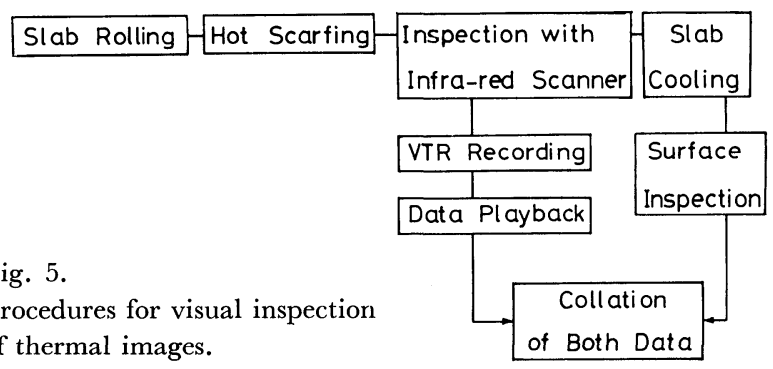




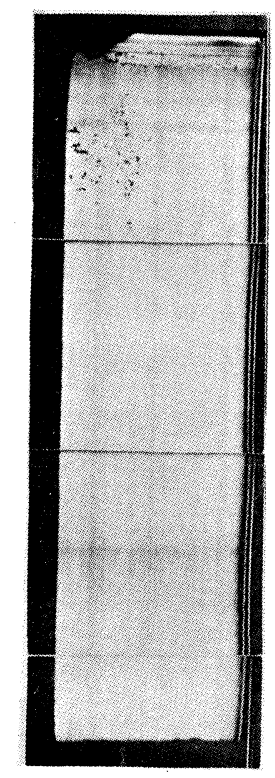

Thermal Image
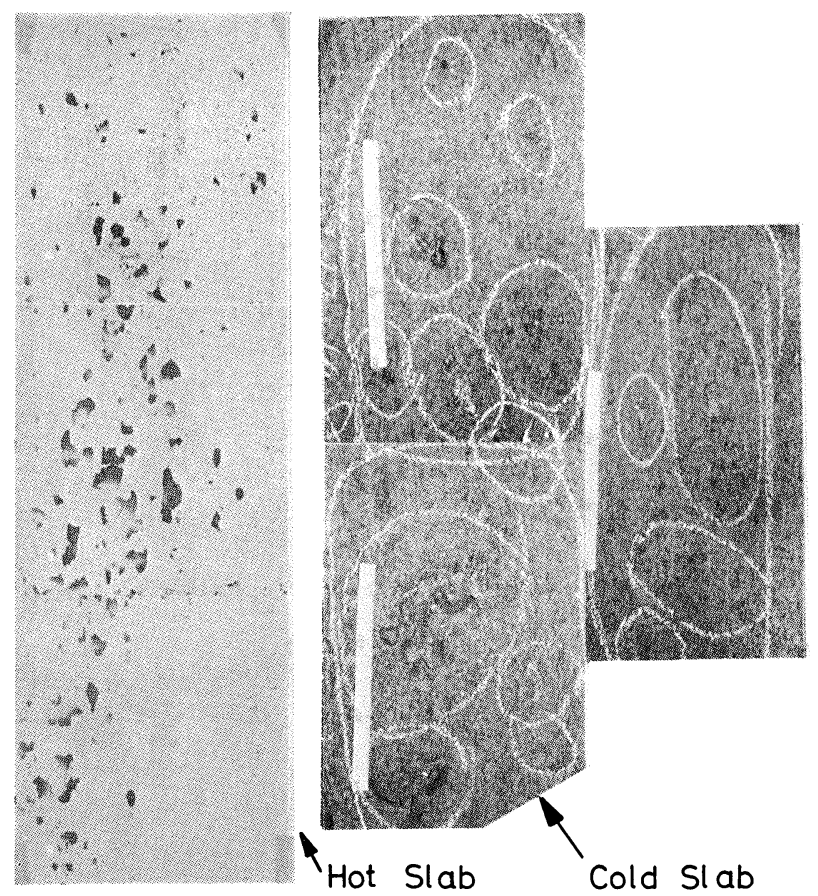

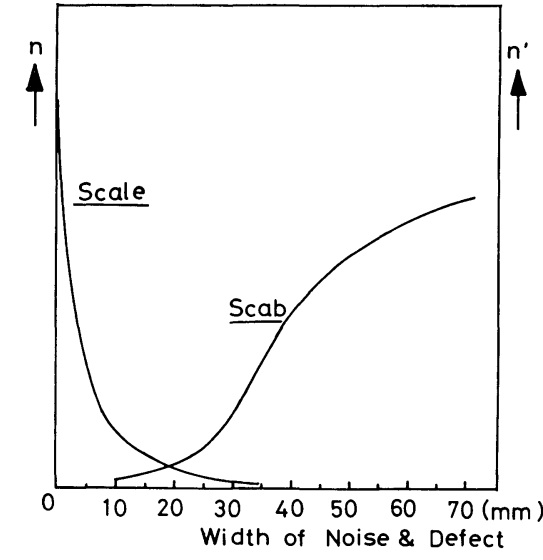

" $n$ " means the number of scales on a slab surface.

" $n^{\prime}$ " means that of scabs (defects).

(The units of $n$ and $n^{\prime}$ are chosen arbitrary but the same.)

Fig. 6. Width distribution of scales and scabs.
Thermal Image: image of a hot slab made with the infrared scanning camera.

Hot Slab : image (partial) of the same hot slab, made with a $35 \mathrm{~mm}$ camera.

Cold Slab : : image (partial) of the same slab after cooling, made with a $35 \mathrm{~mm}$ camera.

Photo. 1. Comparison of three types of a defect image.

the "Cold Slab" image is, "Hot Slab" images were sometimes made to make the collation easier.

Thus it was found that for a slab surface with little scale or water cover the visual detectability of defects was quite high; more than $85 \%$ of scabs, more than $95 \%$ of tong marks, more than $90 \%$ of zig-zag cracks and more than $70 \%$ of longitudinal cracks were detected.

\section{Development of Image Processing Equip- ment}

\section{Quantitative Analysis of Defect Signals}

Since visual inspection of the thermal images made by the scanning camera showed a high defect detectability, the project advanced to the next stage: the development of image processing equipment for automatic defect detection, to replace visual inspection. As a first step in this development, the thermal images were analysed quantitatively, with the aim of determining logic effective in the extraction of defect signals.

The basic unit in the analysis was a "signal scan". (Note: A "signal scan" means in this paper a series of digital or analog signals recorded on one scan in the transverse direction across a slab. Any signal scan can be retrieved from high-speed camera film.) The following features were noted:

Feature 1: There is a strong tendency for defects to produce pulse signals.
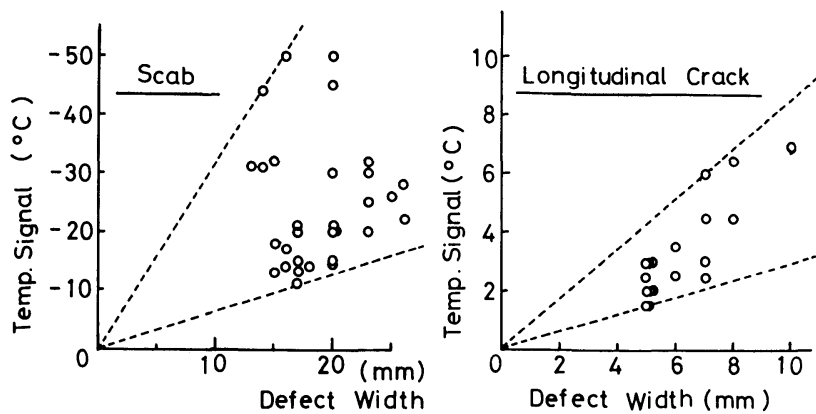

Fig. 7. Relation between the width and the temperature signal of defects.

Feature 2: Scale which has appeared on the slab surface is sometimes difficult to distinguish from scabs. A statistical study, however, showed that scale and scabs could be distinguished fairly well from each other from respect to their width, shown as graphically in Fig. 6.

Feature 3: As shown in Fig. 7, defects such as scabs give "minus" or low temperature signals, which means these defects have a lower temperature than the surrounding normal areas. Defects such as cracks or blowholes, however, give "plus" or high temperature signals, and the temperature difference between " plus" signals and those from normal areas is about one tenth of that of "minus" signals. It should be noted that since no emissivity correction was made, any temperature quoted is an apparent temperature, with an emissivity equal to one.

Feature 4: The ratio of the temperature difference to the defect width varies within a fixed range. This range is peculiar to the kind of defect, as shown in Fig. 7.

Feature 5: Many defects are stretched in the longitudinal direction, being affected by slabbingrolling in that direction. 


\section{Designing of the Image Processing Equipment}

The above features, Features 1 to 5 in the last section, suggested a series of data processing stages as follows:

Extraction 1: Extraction of pulse signals (Feature 1). Extraction 2: Extraction of those signals whose width is larger than a given value (Feature 2).

Accumulation: Longitudinal accumulation of a given number of signal scans (Feature 5).

Extraction 3: Extraction of those accumulated signals whose amplitude is larger than a given value (Features 2 and 3). The two kinds of signals, plus or minus, are extracted with different values.

Extraction 4: Extraction of those signals whose ratio of amplitude to width is less than a given value (Feature 4).

Linkage: Linkage of the signals within a given range to each other (Feature 5).

Extraction 5: Extraction of those linked signals whose width is either larger than a given value or whose length in the longitudinal direction is larger than another given value (Features 2 and 5).

As a compromise between efficiency and flexibility, the image processing equipment was designed to consist of two components: a signal processing unit with little flexibility but a high efficiency and a computer and peripheral equipment with great flexibility due to the programming but with great redundancy. The signal processing unit does the processing stages from Extractions 1 to 3 and the computer does Extractions 4 and 5. The total system is as shown in Fig. 8. The principal specifications of the processing apparatus are listed in Table 2.

Many parameters in the image processing equipment have a range of freedom, as seen in Table 2. For example, the threshold level in Extraction 2 has three values: 1,10 and 20 . This is set to the one that provides optimum detectability for actual defects, as detailed in Section V. 6 .

\section{Data Processing Unit}

1. Signal Flow through the Data Processing Unit

The signal flow through the data processing unit is shown schematically in Fig. 9 (" $\mathrm{H}$ Defect" in the figure means high temperature defect signals and " $\mathrm{L}$ Defect" means low temperature defect signals). The details of the signal flow are explained below:

(1) The signal either from the scanning camera or from a VTR is transmitted to an A/D converter through an interface.

(2) In every digitalized signal scan, a " background variation" is artificially derived by the method described in Section V. 3.2.

Note: A "background variation" means in this paper a smooth amplitude variation along the scanning direction with no pulse signals.

Subtraction of background variation from the signal scan leaves only pulse signals: the " defect signals".

Note: A "defect signal" means hereafter in this paper a signal extracted by the preceding extraction process. A "defect signal", therefore, does not always mean the signal from a real defect.

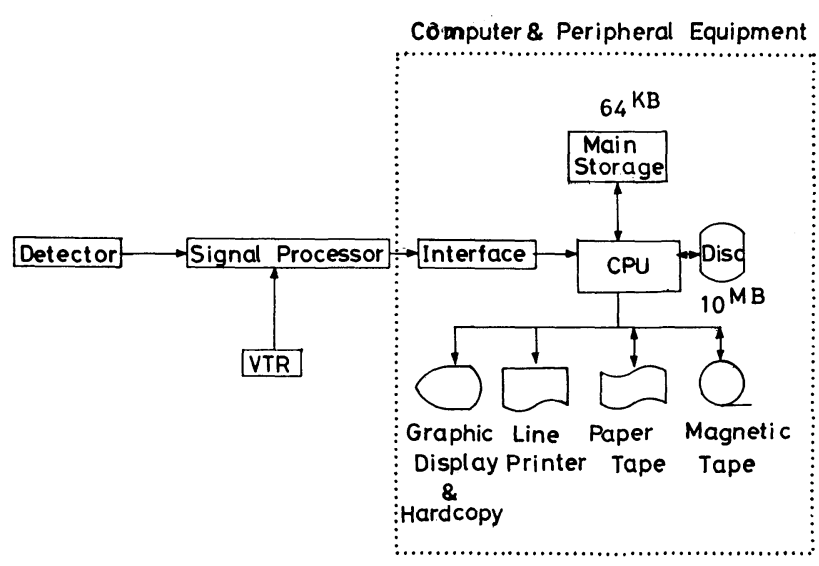

Fig. 8. Design of an image processing apparatus.

The image processing apparatus consists of a signal processor and a computer with its peripheral equipment. The central processing unit (CPU) is linked to a main storage with 64 kilo-bites and also to a disk memory with 10 mega-bites.

Table 2(a). Specifications of the data processing unit.

\begin{tabular}{l|l|l}
\hline \multicolumn{1}{c|}{ Item } & \multicolumn{1}{|c|}{ Range } & \multicolumn{1}{|c}{ Memorandom } \\
\hline Extraction 2 & $1,10,20 \mathrm{~mm}$ & $\begin{array}{l}\text { Only for minus } \\
\text { signals } \\
\text { Detailed in Section } \\
\text { V.3.3. } \\
\text { factor }\end{array}$ \\
$\quad$ Plus & $8,15,30,45,60^{\circ} \mathrm{C}$ & \\
Minus & $30,60,120,180$, & \\
Extraction 3 & $240^{\circ} \mathrm{C}$ & \\
Continuously & changeable & \\
Accumulation & $1,2,5,10,15,20$, & \\
number & 25,30 & \\
Sampling ratio & $1 / 11 / 2,1 / 4,1 / 6$ & \\
\hline
\end{tabular}

1) Sampling ratio. The usual situation is $1 / 1$ (sampling all data scans) for $10 \mathrm{~m} / \mathrm{sec}$ (the conveyance velocity of the hot slab). Another selection $1 / 2$, for example, could simulate a possible situation of $1 / 1$ for $20 \mathrm{~m} / \mathrm{sec}$.

2) The two kinds of signals, plus and minus, can have different values in Extraction 3, Accumulation number and Sampling ratio.

Table 2(b). Specifications of the computer.

\begin{tabular}{l|l}
\hline \multicolumn{1}{c|}{ Item } & $\begin{array}{l}\text { Panafacom PFU-300 with 64 kilo- } \\
\text { bites core memory } \\
\text { Magnetic tape with } I / O \\
\text { Line printer } \\
\text { Paper tape with } I / O \\
\text { Graphic display } \\
\text { Extraction 4 } \\
\text { Linkage } \\
\text { Extraction 5 } \\
\text { (Any level or range is acceptable } \\
\text { in the programming) } \\
\text { Line printer } \\
\text { Two dimensional presentation } \\
\text { of defects } \\
\text { Listing of the position of defects } \\
\text { Graphic display } \\
\text { With or without data processing } \\
\text { in the computer }\end{array}$ \\
\hline
\end{tabular}

(3) Minus defect signals whose width is less than a given value are eliminated, on the assumption that 


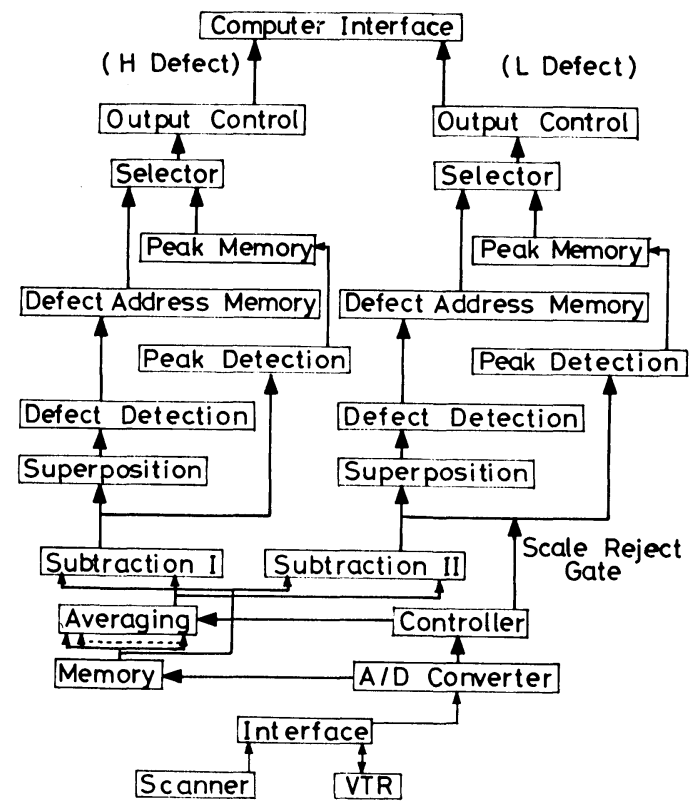

Fig. 9. Extraction of defect signals in the data processing unit.

these signals are not from defects but from scale.

(4) A given number of signal scans are accumulated in such a way that those signals which have a common longitudinal position and adjacent transverse position are combined to make up an accumulated signal scan. That means, for example, that the signals from the $\mathcal{N}$-th scan to $(\mathcal{N}+9)$-th scan make up one accumulated signal scan, if the accumulation number is ten. Similarly, the signals from the $(\mathcal{N}+$ $10)$-th to the $(\mathcal{N}+19)$-th scans make up another accumulated scan.

(5) In every accumulated signal scan, the starting signal and the end signal of each continuous sequence which exceeds a given value are detected in the way described in Section V. 3.3. At the same time, the highest amplitude of the signal between these two points before accumulation is counted.

(6) The addresses of the starting signal and the end signal and the highest amplitude are stored as three words, each of which consists of sixteen bits. These are transferred to main storage in the computer and then are subjected to further data processing. Figure 9 shows how the defect signals are derived or extracted by the data processing unit.

\section{Derivation of the Background Variation}

One of the most successful attempts made by the authors is the application of a floating threshold level method to the derivation of a background variation. Theoretical comparison of this method with two other conventional methods, the high pass filtering method and the modified low pass filtering method, showed that this would be best.

The high pass filtering method, shown as HPF in Fig. 10, is the simplest of the three, because the relatively higher frequency components of a signal scan pass through the filter. Since most of the signals from defects are expected to contain high frequency components, they should be filtered out. There is, however, a crucial fault associated with this method;

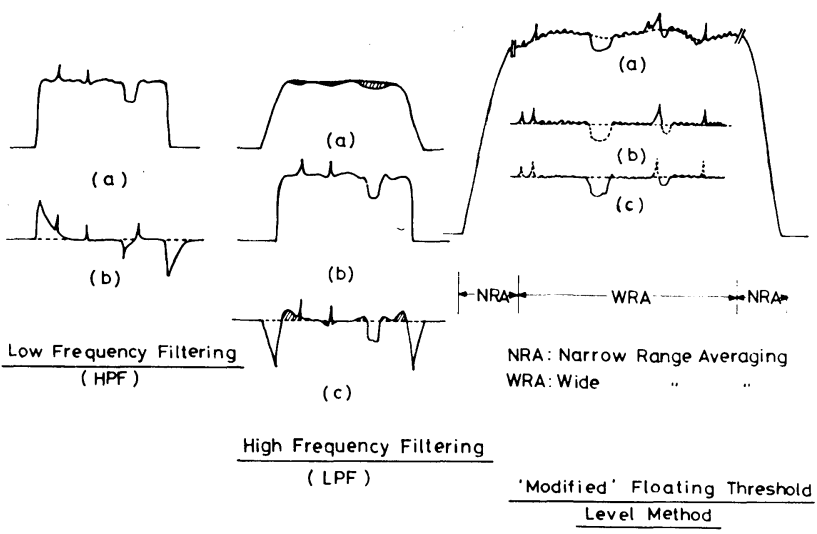

HPF: (a) original signal profile.

(b) signal profile through HPF, causing false defect signals at both ends.

LPF: (a) signal profile being smoothed through LPF.

(b) original signal profile.

(c) signal profile after subtraction of (a) from (b) causing false defect signals at both ends.

Modified floating threshold level method:

(a) original signal profile.

(b) plus defect signals (on the solid line).

(c) minus defect signals (on the solid line). no false signals on (b) or (c)

Fig. 10. Three methods for the creation of a back ground variation.

when this: method is applied to a trapezoid scan waveform, as is the one here, the vicinity of the edge of the slab produces false background signals. A defect near the edges, if there is one, may not be detected by this method, as illustrated in Fig. 10.

The modified low pass filtering method, shown as LPF in Fig. 10, subtracts from a signal scan the same scan which has been passed through a low pass filter. This method has the same fault as the HPF method, in that defects near the edges are hard to detect, as shown in Fig. 10. Moreover, it has a further fault in that it tends to create at both edges of a defect signal fault-defect-signals of the opposite sign, because of residual effects of the defect signal, as shown by the slant lines for the LPF in Fig. 10.

The idea for the application of the floating threshold level method derived from the advantageous fact that the signal is digitalized. This method consists of three steps: averaging of the amplitudes of a number of successive signals (Step 1); setting the background amplitude of the signal positioned in the middle of the averaging range as the average value (Step 2); and shifting the averaging range one step and repeating (Step 3).

Considering the signal features of a hot slab, two modifications were made:

Modification 1:

The slab width is divided into two types of zone: the zones near the edge (edge zone), and the remaining zone (central zone). The proportions of the two zones are decided by the slope of the amplitude change along the scanning direction. The averaging range in the edge zone is small, for example, five signals, so as to follow the rapid temperature changes 
near the edge. The averaging range in the central zone is large, for example, sixty five signals, due to the slow change of temperature.

\section{Modification 2:}

Every incoming signal is compared with the averaged background value for the preceding averaging range. If the difference between the amplitude of the signal and the average value is less than a given value, the amplitude of the signal positioned in the middle of the new averaging range is replaced by the average value. If not, it is replaced by the one in the preceding averaging area. This modification prevents sharp pulse signals from affecting the derivation of a smooth background variation.

Photograph 2 shows two examples, in each of which a signal scan is compared with the corresponding background variation derived by this method. It should be noted that those background variations are almost ideal in terms of fidelity near the edges. Photograph 3 shows three examples in each of which a signal scan is compared with the new signal scan created by subtracting the corresponding background variation from the signal scan. After this subtraction only pulse signals remain, that is, defect signals: in the upper and middle examples minus defect signals are shown, and in the lower example plus signals.

\section{Extraction of Signals Taking into Account Signal Amplitude}

Noting that plus defect signals have different amplitudes from minus defect signals (Feature 3), the extraction takes into account the signal amplitude $(E x$ traction 3) in two stages:

Stage 1: The two kinds of defect signals are amplified (normalized) using different normalization factors, as shown in Table 2(a).

Stage 2: After signal accumulation, the normalized signals, whether they are plus or minus, are subjected to amplitude extraction using a common threshold level.

The advantage of such two stage processing is that, as can be seen from the example below, the crucial separation of defect signals from others (noise) is always possible, independent of the amplitude of the signals. Giving an example will make it clear what is done in the two stages: Example
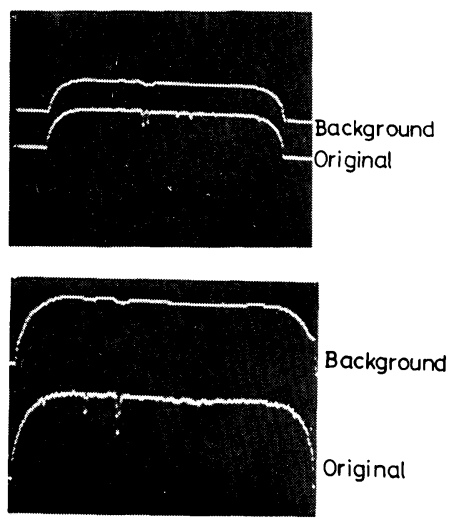

Photo. 2.

Background variation derived by the modified floating threshold level method.

\begin{tabular}{l|ccccc}
\hline $\begin{array}{c}\text { Kind } \\
\text { of } \\
\text { defect }\end{array}$ & Amplitude $\begin{array}{c}\text { Normali- } \\
\text { zation } \\
\text { factor } \\
\text { (Stage 1) }\end{array}$ & $\begin{array}{c}\text { Norma- } \\
\text { lized } \\
(\%)\end{array}$ & $\begin{array}{c}\text { Threshold } \\
\text { (Stage 2) }\end{array}$ & Result \\
\hline plus & $4.3^{\circ} \mathrm{C}$ & 8 & 54 & 52 & defect \\
plus & 4.0 & $\begin{array}{l}\text { same as } \\
\text { above }\end{array}$ & 50 & $\begin{array}{l}\text { same as } \\
\text { above }\end{array}$ & noise \\
minus & 35 & 60 & 58 & 54 & defect \\
minus & 30 & $\begin{array}{c}62 \\
\text { same as } \\
\text { above }\end{array}$ & 50 & $\begin{array}{l}\text { same as } \\
\text { above }\end{array}$ & noise \\
\hline
\end{tabular}

\section{Computer and Peripheral Equipment}

The computer used is specified in Table 2(b) and the signal flow is shown in Fig. 11. The peripheral data processing that the computer undertakes is listed below:

(1) Extraction of those signals whose ratio of amplitude to width is less than a given value (Extraction 4).

(2) Artificial reconstruction of defect patterns by linking those signals which are close to each other in space (Linkage). In other words, any defect signals within a given distance of a common defect signal are processed as belonging to the same defect on the slab.

(3) Extraction of those linked signals whose width is either larger than a given value or whose longitudinal length is larger than another preset value (Extraction 5).

(4) Output of defect signals by means of a line printer or graphic display.

This processing has nothing to do with so-called "pattern recognition", which involves more complicated data processing than that listed above, due to apprehension that such pattern recognition would consume so much processing time that the resultant defect information, however correct, would be too late for the following spot scarfer operation.

The signal is processed through the computer in two different ways, real time processing or non-real time processing (off-line processing), as explained in Fig. 11.
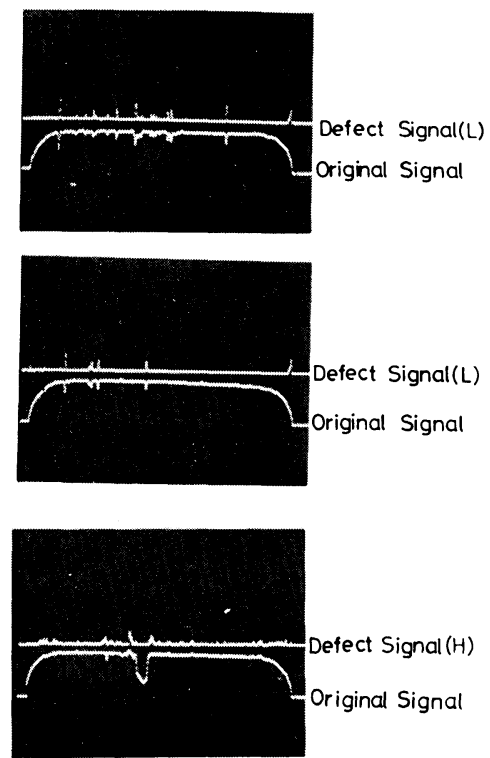

Photo. 3

Subtraction from a signal scan of the corresponding background variation. 


\section{Setting the Adjustable Parameters to Optimum Values}

As mentioned earlier, many parameters in the image processing apparatus have a range of freedom. These have been set at their "optimum" values through further experiment.

Note: An "optimum" value means in this paper the one which yields the nearest pattern to the photographic image of the corresponding defect taken when the slab is cooled.

Several hundred hot slabs produced by the No. 3 slabbing mill at Chiba Works were used for the ex-

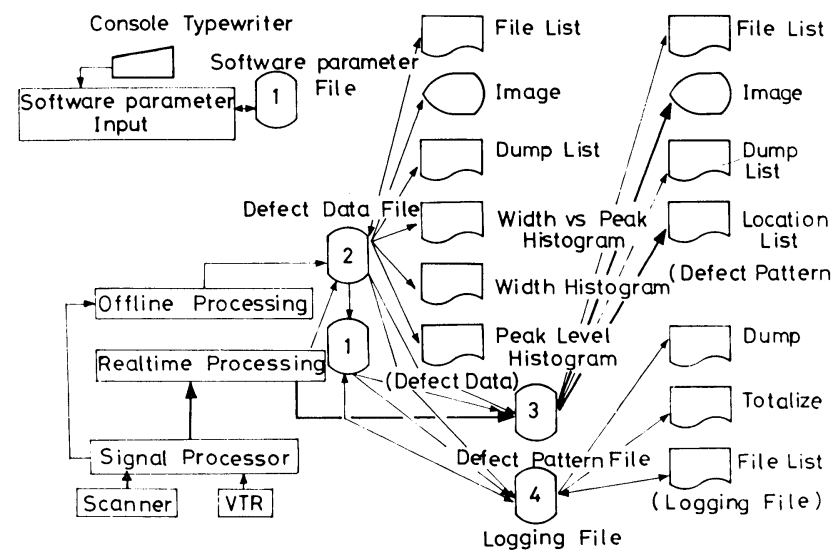

Fig. 11. Signal flow through the computer and the peripheral equipment. periment; the infrared images of these slabs were recorded on the VTR and subsequently retrieved for non-real time processing on the computer to find the optimum values.

The two examples in Photos. 4 and 5 give an idea of the procedure. Photograph 4 shows that one combination of parameters emphasizes scabs and another combination a zig-zag crack, although the input defect information used was the same. Photograph 5 illustrates the effect of the threshold level in Extraction 3 on the appearance of scabs; decreasing the threshold level results in over-detection so that low level noise signals are extracted as defect signals. Another study demonstrated that increasing the accumulation number improves the $S / \mathcal{N}$ ratio but worsens the ability to reproduce defect patterns.

As can be seen in Table 3, sixty four combinations for minus defect signals and thirty two for plus signals were checked. As a result, the following combinations were found to yield the optimum results:

$\begin{array}{lll} & \text { Minus } & \text { Plus } \\ \text { Normalization factor } & 60^{\circ} \mathrm{C} & 15^{\circ} \mathrm{C} \\ \text { Accumulation number } & 10 & 10\end{array}$

Threshold level

within the range 10 to 30

Photographs 5 to 7 show two examples in which the parameters were set at optimum. It should be noted
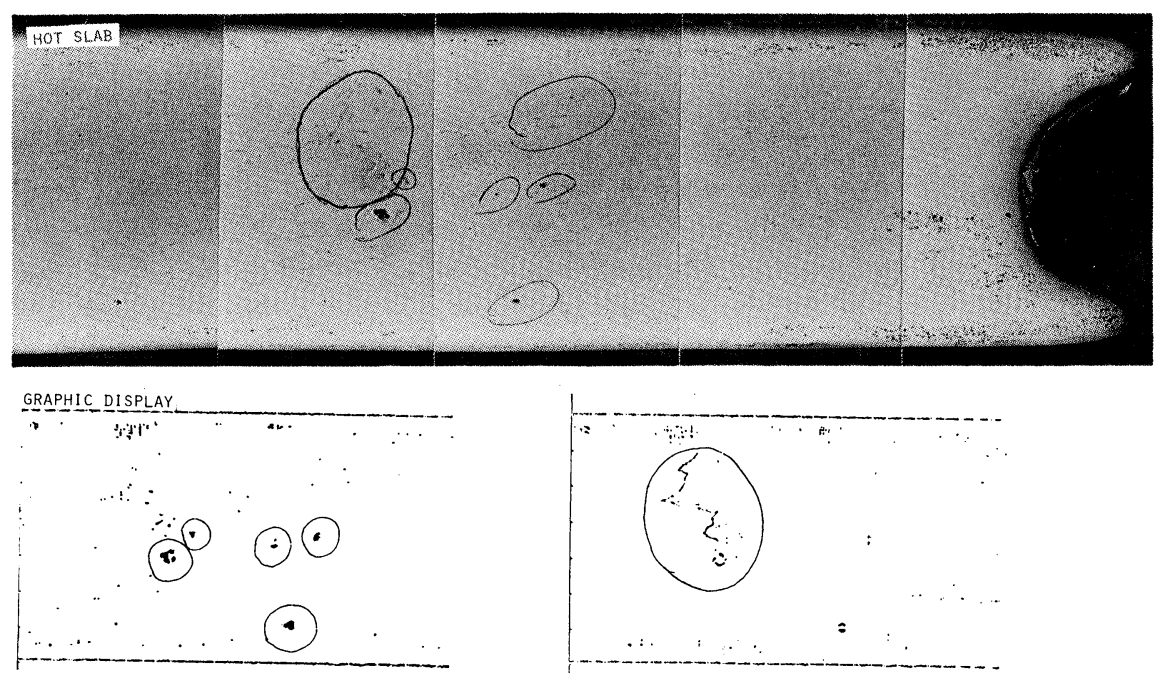

Photo. 4.

Effects of signal processing parameters on the defect data image ('scab' and 'zig-zag crack').

The photographic image of a hot slab ("Hot Slab") shows a zig-zag crack, marked with the largest circle, and five scabs marked with smaller circles. In the left hand "Graphic Display" the five scabs are easy to recognize, but the zig-zag crack is hard to distinguish. In the right hand "Graphic Display", however, the zig-zag crack is easy to recognize, but the scabs are hard to see.

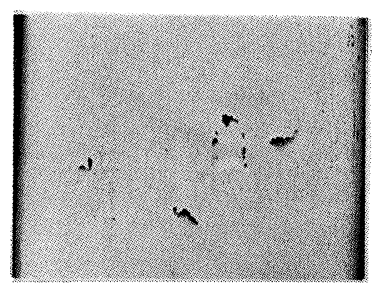

Hot Slab

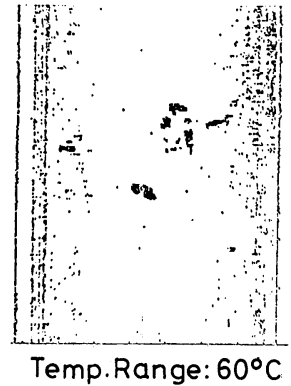

Filter: $10 \mathrm{~mm}$

Sup.Position: 10

Th. Value: 20

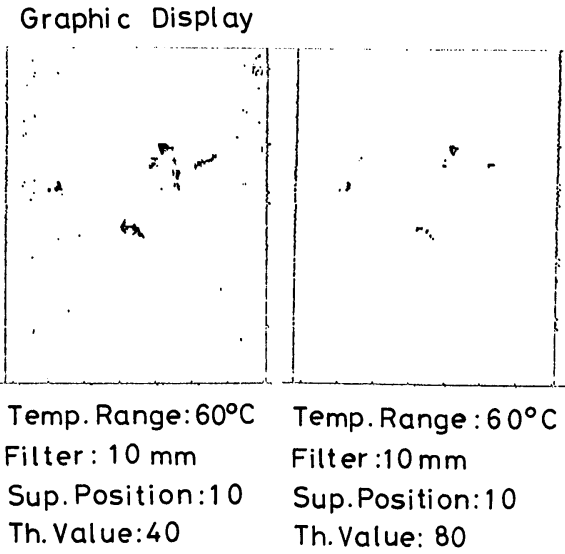

Photo. 5.

Effect of threshold value on the defect data image ('scab').

Decreasing the threshold value can result in over-detection, as shown in the left hand "Graphic Display" (threshold value 20). With this threshold value not only scabs but also scale near the edges are detected as defects. 
that, as shown in Photo. 7, the linkage processing in the computer is able to reproduce the pattern of longitudinal cracks quite faithful to the originals.

\section{Evaluation of the Defect Detection System as Developed}

The whole detection system was evaluated in terms of defect detection. The results of this evaluation were:

Table 3. Parameters for the data processing unit.

\begin{tabular}{l|l}
\hline \multicolumn{1}{c|}{ Parameter } & \multicolumn{1}{|c}{ Range } \\
\hline Extraction 2 & $1,10 \mathrm{~mm}$ (only for minus signals) \\
Accumulation number & $5,10,20,30$ \\
Normalization factor & \\
$\quad$ Minus defect signal & $60,120^{\circ} \mathrm{C}$ \\
Plus defect signal & $15,30^{\circ} \mathrm{C}$ \\
Extraction 3 & Four levels \\
\hline
\end{tabular}

(1) Defects such as scabs are detected nearly perfectly in both their shapes and locations on the slab.

(2) Scale on a slab surface can produce false defect signals; data processing applied with the parameters set at optimum can not eliminate all the signals from scale.

(3) The logic used for linkage of the defect signals close to each other reproduces defect patterns nearly perfectly, although the logic is quite simple.

(4) Longitudinal cracks are not always detected, the reason of which is that the amplitude of the false-defect signals mentioned above in (2) is sometimes comparable with that of signals from longitudinal cracks.

(5) The background variation created by the modified floating threshold method is almost ideal, in that substracting from a signal scan the corresponding background variation leaves only pulse signals, most of which are from the defects on the slab. It has turned out, however, that this method itself is also not quite free from the creation of the same kinds of
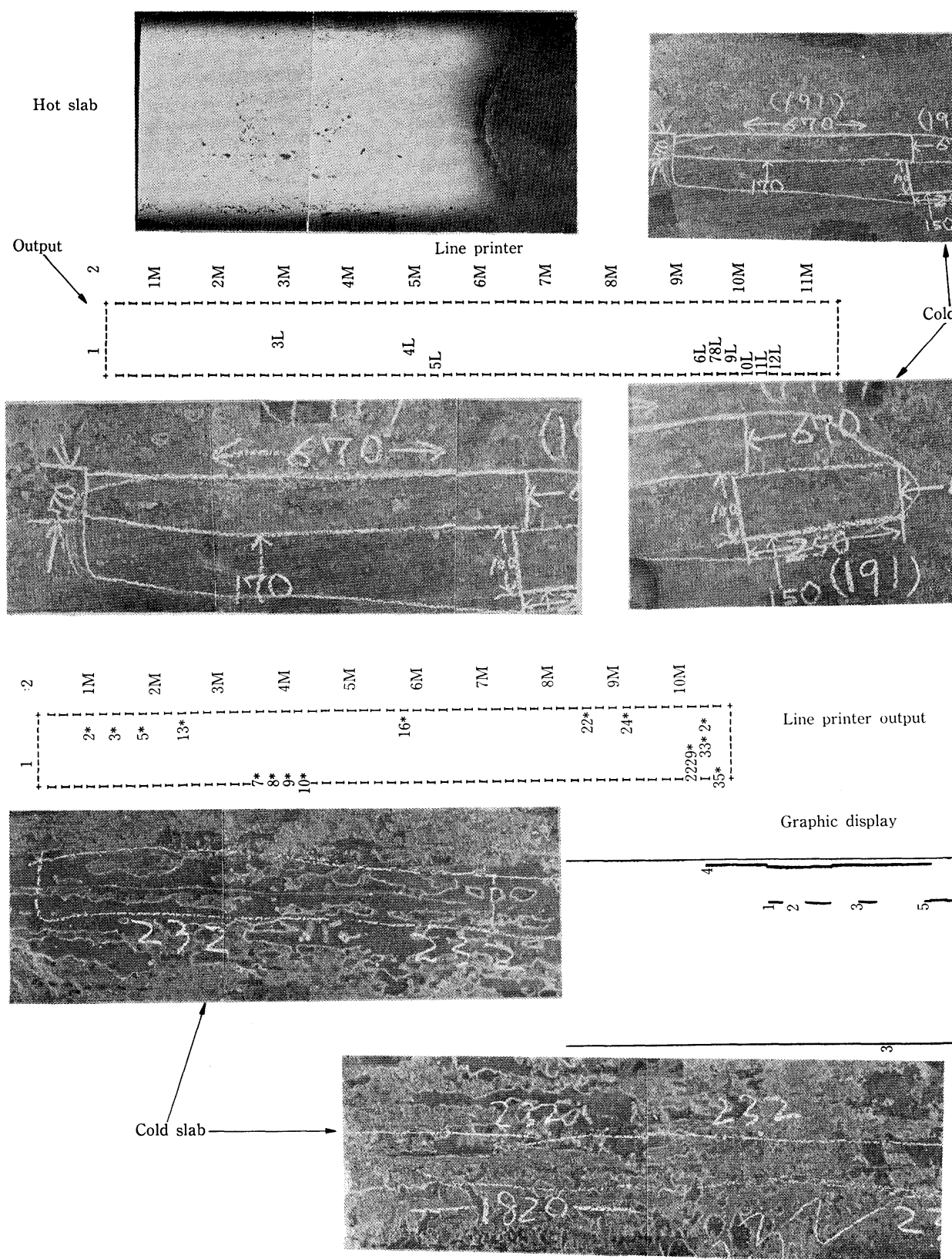

Photo. 6.
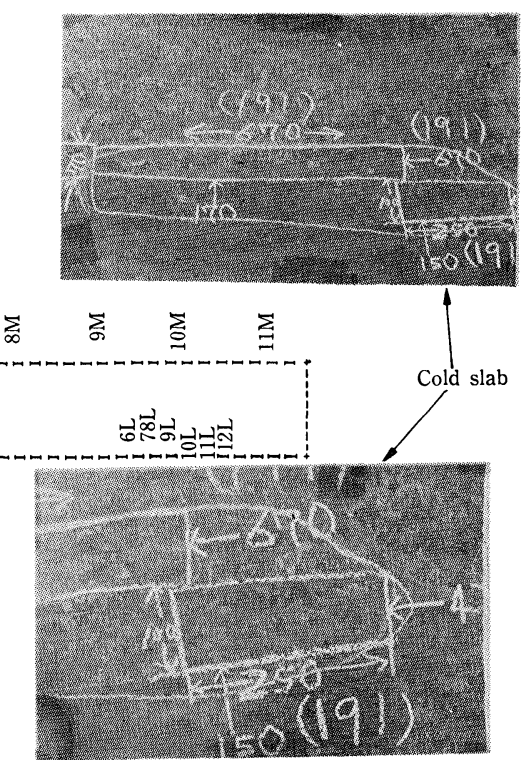

Line printer output

Graphic display

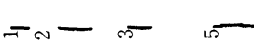

Defect pattern final output ('scab').

The "Line Printer Output", the final output of the image processing equipment, shows that this surface has twelve defects located as indicated. The defects are numbered from $6 \mathrm{~L}$ to $11 \mathrm{~L}$ (L means a low temperature signal) can also be recognized in the top left "Hot Slab", a photographic image of the same hot slab. They can also be recognized in the top right "Cold Slab", a photographic image of the same slab after cooling. The two pictures at the bottom are enlarged portions of the "Cold Slab", for a better understanding (5M, for example, in "Line Printer Output" means the distance from the top of the slab.).

Photo. 7.

Defect pattern final output ('longitudinal crack').

The "Line Printer Output" shows that this surface has about thirty five defects $(*$, for example $3^{*}$, means simultaneous presentation of both low temperature signals and high temperature signals.). Longitudinal cracks on "Graphic Display" correspond to the defects numbered 2, 3 and 5 on "Line Printer Output". The two "Cold Slabs" show the same cracks, which can be recognized as the thin lines between the white chalk-lines. 
false defect signals that are created by the high pass filtering method or the modified low pass filtering method, although the occurrence of such false signals is the least for this method among the three. This means that for some high amplitude or sharp pulse signals this method can still give rise to false signals on both sides of the defect.

\section{Summary}

An automatic surface detection system for hot ingot-casting-slabs has been developed in the following stages:

(1) Among several possibilities, the thermal image of a hot slab provided by an infrared scanning camera was evaluated as being the best for inspection.

(2) The best filter and detector size combination for the scanning camera was selected from the given possibilities. It was found that a scanning camera using this combination can give thermal images of hot hot slabs on which most defects can be visually recognized.

(3) Image processing equipment, to replace visual inspection, was designed, consisting of two components: a signal processing unit and a mini-computer. Several kinds of data processing were built into the equipment for the extraction of defect signals.

(4) Many parameters in the equipment were given a range of freedom and adjusted to fixed values that provided the optimum detection capability for real defects.

It was then concluded that the detection system could enable automatic on-line detection, if the following items were added or improved:

(1) A descaling device, a mechanical remover of scale on a hot slab, is installed before the detection system.

(2) Improvement in the logic of the floating threshold level method succeeds in eliminating false defect signals resulting from some pulse signals.

(3) The three other sides of the hot slab are also inspected by the same detection system.

\section{Future Plans}

\section{Coupling with a Spot Scarfer}

The project is now at the stage of coupling the surface defect detection system to an automated spot scarfer: the method of conveying defect information to the spot scarfer is under discussion.

\section{Automatic Surface Defect Detection in Continuous Cast- ing Slabs}

The currnt etrend to increasing production of continuous-casting-slabs has set to take prompt action in the development of a surface defect detection sys- tem for these slabs. The techniques acquired in the development of the detection system for ingot-castingslabs, especially the data processing for defect signal extraction, will be of great use in defect detection in continuous-casting-slabs.

However, since the surface defects of continuouscasting-slabs are not quite the same as those of ingot-casting-slabs, the detection method applied to ingot-casting-slabs, that is the thermal image inspection method, is not necessarily the best for continuous-casting-slabs. There are a number of alternative possibilities under discussion:

(1) Wavelength: infrared or visible

Powder or oscillation marks on a continuous-casting-slab may cause the radiation from the slab, most of which lies in the infrared range, to fluctuate significantly. This fluctuation makes the infrared scanning camera method less advantageous.

(2) Active or Passive

One of the disadvantages of the passive method, which was revealed in the development of the ingotcasting-slab inspection system is that the defect signals detected by the passive method have both plus or minus values, which makes the following data processing more complicated than the active method would. (3) Detection device: Color ITV, CGD camera or multi-linear image sensor

Several sensors have recently come into practical use, such as the charge-coupled device (CCD) camera and the multi-linear image sensor. The detection capability of these sensors is now comparable with that of color industrial television (ITV).

(4) Detection point: the delivery side of the pinch rolls for the middle of the two rolls on an upper roller apron

Although no final decision has been made, the delivery side of the pinch rolls is considered more desirable, as explained in Chapter II.

(5) Number of sport scarfers: one or two

As also mentioned in Chapter $I I$, it is proposed to inspect the surface defects on a continuous-casting-slab twice. The double inspection poses the question of whether two spot scarfers are necessary or not: one scarfer after each detection device or a single scarfer after the secondary detection device. The two possibilities are being discussed.

At two major plants of the Company, Chiba Works and Mizushima Works, experiments are now under way to evaluate these alternatives.

\section{REFERENCES}

1) H. Kitagawa, A. Fujii, S. Miyake and K. Kurita: Tetsu-toHagané, 64 (1978), S743.

2) H. Kitagawa, A. Fujii, S. Miyake and K. Kurita: Tetsu-toHagané, 65 (1979), S247. 